\title{
Comparative Study of Outcomes Following Laparoscopic Versus Open Peritoneal Dialysis Catheter Insertion at a Tertiary Care Centre
}

\author{
(D) Raghav Talwar1, (D) Aditya Jha1, (D) Govindaiah Madhu1, (D) Neha Singh2, (D) Gagandeep Singh1 \\ ${ }^{1}$ Army Hospital (Research \& Referral), Clinic of Urology, New Delhi, India \\ ${ }^{2}$ Army College of Medical Sciences, Community Medicine, New Delhi, India
}

\section{What's known on the subject? and What does the study add?}

Currently, different surgical techniques are in practice for peritoneal dialysis catheter placement with varied success and complication rate. Literature describes a failure rate of $10-35 \%$ when catheters are placed via open technique and $2.8-13 \%$ via laparoscopic technique. Despite open technique being most frequently used, laparoscopic procedures have shown superiority by reducing morbidity, length of hospital stay, postoperative pain and shorter convalescence. In the existing literature, there is no consensus about the preferred operative technique for peritoneal dialysis catheter insertion. The study sheds further light on the advantages and drawbacks of laparoscopic peritoneal dialysis catheter insertion and compares it to the conventional technique of open continuous ambulatory peritoneal dialysis catheter insertion.

\begin{abstract}
Objective: Continuous ambulatory peritoneal dialysis is a common treatment mode in patients with end-stage renal disease. Various insertion techniques of continuous ambulatory peritoneal dialysis catheters have been described in the literature, including percutaneous, open and laparoscopic techniques, with no consensus about the preferred operative technique.

Materials and Methods: Between August 2016 and March 2018, 50 patients undergoing catheter insertion were randomised to insertion by either the open technique or laparoscopy. The demographic, preoperative and postoperative profiles of these patients were recorded and patients were followed up for six weeks postoperatively. The Pearson chi-square test was used to compare the results with historical controls. Fisher's Exact test was used to assess the incidence of surgical complications. The significance level was set at 0.05 .

Results: A comparison between the two groups indicated that the only significant difference was in postoperative pain on postoperative evening, lower in the laparoscopic group and statistically significant $(p<0.05)$. There was no statistically significant difference in catheter tip migration, catheter exit-site infection, catheter-associated peritonitis or catheter functional status between the two techniques.

Conclusion: The laparoscopic technique does not provide any additional advantage than the open technique in patients undergoing continuous ambulatory peritoneal dialysis catheter placement.
\end{abstract}

Keywords: Continuous ambulatory peritoneal dialysis catheter, comparison, open surgery, laparoscopic

\section{Introduction}

Peritoneal dialysis (PD) is an effective treatment for end-stage renal disease (ESRD). The key to successful PD is the presence of a well-functioning dialysis catheter, defined as one that facilitates free dialysis solution inflow and outflow. However, several complications, such as inflow and outflow obstruction, peritonitis, exit-site infections, leakage and catheter tip migration, can lead to loss of peritoneal access.
Currently, different surgical techniques are used for catheter placement with varied success and complication rates. The literature describes a failure rate of 10\% - 35\% when catheters are placed via open the technique and $2.8 \%-13 \%$ via the laparoscopic technique. Although the open technique is most frequently used, laparoscopic procedures have shown superiority by reducing morbidity, length of hospital stay, postoperative pain and shorter convalescence (1). In the existing literature, there is no consensus about the preferred operative technique

Correspondence: Gagandeep Singh MD, Army Hospital (Research \& Referral), Clinic of Urology, New Delhi, India

Phone: +919764610900 E-mail: gagan15582@yahoo.co.in ORCID-ID: orcid.org/0000-0002-3567-5353

Received: 16.06 .20

Accepted: 30.08 .20

Cite this article as: : Talwar R, Jha A, Madhu G, Singh N, Singh G. Comparative Study of Outcomes Following Laparoscopic Versus Open Peritoneal Dialysis Catheter Insertion at a Tertiary Care Centre. J Urol Surg 2021;8(1):40-45.

${ }^{\circ}$ Copyright 2020 by the Association of Urological Surgery / Journal of Urological Surgery published by Galenos Publishing House. 
for PD catheter insertion. The hypothesis of this study was that the laparoscopic PD catheter insertion procedure leads to a lower incidence of catheter malfunctioning at six weeks postoperatively.

\section{Materials and Methods}

We conducted a randomised controlled study at our hospital between August 2016 and March 2018. After obtaining institutional ethical committee approval (Institutional Ethics Committee, Army Hospital ( $R$ \& $R$ ), Delhi Cantt, IEC Regn no: 93/2016), all patients for PD were included in this trial after obtaining written informed consent. Random numbers were generated using the RAND function of MS Excel. Patients were divided into two equal groups. The results were kept in serially numbered, sealed opaque envelopes. These envelopes were kept with a third person (Head Clerk) at the hospital. Once the patient was enrolled, a call was made to the third person to ascertain the group. Both surgeon and patient were informed about the technique only on the morning of the surgery. All procedures were performed by consultants having a similar experience with vancomycin injection as antibiotic prophylaxis under general anaesthesia.

Patient's with a body mass index (BMI) $>35 \mathrm{~kg} / \mathrm{m}^{2}$, age $<18$ years and those unfit for general anaesthesia were excluded.

The primary objective was to determine a better surgical placement procedure to minimise the incidence of catheter malfunction at six weeks postoperatively.

We also wanted to assess if the use of the laparoscopic insertion technique reduces the rate of surgical complications, surgical mortality, leakage, catheter tip migration, catheter-related readmissions, exit-site infections, peritonitis and postoperative pain.

\section{Surgical Interventions}

\section{Laparoscopic Technique}

Preoperatively, the catheter's exit-site was jointly marked by the surgeon and patient and was sited well above the belt. General anaesthesia and antibiotic prophylaxis (vancomycin, $1000 \mathrm{mg}$ IV) were administered. After cleaning (chlorhexidine disinfection) and draping, the patient was put in the Trendelenburg position. A 10-12 mm port was placed supraumbilically using the open technique and pneumoperitoneum was created.

Using a 30 degrees camera, the peritoneal cavity was inspected. Adhesiolysis was performed wherever necessary. A doublecuffed Swan Neck Tenckhoff dialysis catheter was placed on the patient's abdomen, to determine the best entry and exit points. Subsequently, a small incision was made at the entry point. Using an $8 \mathrm{~mm}$ trocar, a subcutaneous tunnel was created. The trocar was then introduced into the peritoneal cavity. A catheter was then introduced with a stylet, without twisting the catheter around. If necessary, an additional $5 \mathrm{~mm}$ trocar was inserted to enable securing the catheter in the correct position and the catheter tip was placed in the pouch of Douglas. The stylet and $8 \mathrm{~mm}$ trocar were subsequently removed. The distal cuff of the catheter remained just outside the peritoneum. The peritoneal cavity was desufflated. The free inflow and outflow were tested with at least $500 \mathrm{cc}$ of saline with the patient in the neutral position. Then, the balloon trocar was removed. The subumbilical fascia was closed with vicryl round body 3-0 and the skin was closed using Monocryl.

\section{Open Technique}

The preoperative measures were the same. A 4-5 cm transverse incision was made two finger breadths below the umbilicus. Then, the anterior rectus fascia was opened, the muscles were split and the dorsal rectus fascia was opened. The surgeon ensured that the surrounding peritoneum was free of adhesions with his finger. Preferably, the os pubis was felt. The catheter was then introduced as described above and the tip was placed in the pouch of Douglas/rectovesical pouch. Inflow and outflow testing were done as described above. The peritoneum and fascia were closed with a purse-string suture using PDS 3-0. The catheter's proximal end was brought out from a point along the catheter's natural curve, ensuring that the proximal cuff was far enough from the exit point.

In the immediate postoperative period, patients had a plain abdominal X-ray abdomen after 24 to 48 hours of catheter placement (after the passage of stools) to document the catheter tip's correct position. Postoperatively, patients were also asked to complete different standardised questionnaires to evaluate pain [visual analogue scale (VAS) score]. The patients were reviewed on postop evening (D+0), D+1, D+2, D+3 and $\mathrm{D}+7$ to assess pain scores as per VAS. PD training was started in both groups after 14 days postoperatively. They were followed till six weeks postoperatively to assess the catheter's functional status and document any complications.

\section{Statistical Analysis}

Categorical variables were presented as numbers (percentages). Continuous variables were presented as medians (ranges). Categorical variables were compared using the chi-square test. Continuous variables were compared using the Mann-Whitney $\mathrm{U}$ test. All analyses were conducted using SPSS (version 17.0, SPSS Inc., Chicago, USA). A p-value $<0.05$ was considered significant.

\section{Results}

Total of 50 patients who underwent continuous ambulatory peritoneal dialysis (CAPD) catheter placement at our institute 
between August 2016 and March 2018 were recruited. They were randomised equally, with 25 patients undergoing laparoscopic catheter placement (group 1) and 25 undergoing open catheter insertion (group 2). Patients were reviewed on $D+0, D+1, D+3$, $D+7$ and $D+6$ weeks to respond to various study parameters and check the catheter's functional status (Table 1).

Group 1 comprised 18 (72\%) males and seven females (28\%), whereas group 2 comprised of 19 (76\%) males and six (24\%) female patients $(p=0.747)$. Regarding age distribution, the mean age in group 1 was $50.88+/-7.59$ yrs and in group 2 it was $55.12+/-8.54$ yrs $(p=0.054)$. The mean BMI in group 1 was $25.9+/-1.41 \mathrm{~kg} / \mathrm{m}^{2}$ and in group 2 it was $25.4+/-1.2 \mathrm{~kg} / \mathrm{m}^{2}$ $(\mathrm{p}=0.177)$. In group $1,10 / 25(40 \%)$ whereas in group $2,3 / 25$ $(12 \%)$ had a history of previous abdominal surgery $(p=0.024)$. Some patients had undergone previous CAPD catheter insertion, which had been removed for various indications like outflow failure, CAPD peritonitis and other issues. There were a total four such patients who were equally divided in the two arms of two each (Table 2).

The VAS pain score on D+0 (postop evening) was 8 in group 1 and 8.72 in group $2(p=0.006)$. There was no difference in the VAS pain scores in the two groups on D+1, D+2, D+3 and D+7. Patients were followed up in the Nephrology and Urology OPD for catheter training, dialysis or in the event of any complication noticed by the patient or caregiver while administering CAPD.

\begin{tabular}{|c|c|c|}
\hline & Laparoscopic & Open \\
\hline \multicolumn{3}{|c|}{ Gender distribution } \\
\hline Male & 18 & 19 \\
\hline Female & 7 & 6 \\
\hline \multicolumn{3}{|c|}{ Age distribution } \\
\hline $35-45$ & 5 & 3 \\
\hline $46-55$ & 13 & 10 \\
\hline $56-65$ & 6 & 9 \\
\hline$>65$ & 1 & 3 \\
\hline \multicolumn{3}{|l|}{ BMI } \\
\hline $20-23$ & 2 & 1 \\
\hline $23.1-25$ & 2 & 8 \\
\hline $25.1-27$ & 14 & 13 \\
\hline$>27$ & 7 & 3 \\
\hline \multicolumn{3}{|c|}{$\mathrm{H} / \mathrm{O}$ previous abdominal surgeries } \\
\hline Yes & 10 & 3 \\
\hline No & 15 & 22 \\
\hline \multicolumn{3}{|c|}{ Previous CAPD insertion } \\
\hline Yes & 2 & 2 \\
\hline No & 23 & 23 \\
\hline
\end{tabular}

A total of 20 patients, 10 each in both groups (40\% of patients in either group) were readmitted for catheter-related complications.

Five patients had pericatheter leakage during the study period. Of these, two were from group 1 ( $8 \%$ of patients) and three were from group 2 ( $12 \%$ of patients) ( $p=1.0)$.

There was only one mortality in the entire study population, which was in group 1. Catheter tips were found to have migrated out of the true pelvis in seven cases in total (detected by performing an abdominal $\mathrm{X}$-ray). Of these, three cases were in group 1 (12\% of patients) and four were in group 2 (16\% of patients) ( $p=1.0)$.

Of the total readmissions for CAPD catheter-related complications, 10 were because of catheter-related peritonitis. Four occurred in group 1, whereas six occurred in group 2 $(p=0.48)$.

Four patients had catheter site infections. Of these, three were in group 1 (12\% of patients), whereas one was in group 2 (4\% of patients) $(p=0.1)$.

At the six-week postoperative follow-up, two patients in group 1 (8\% of patients), whereas three patients in group 2 (12\% of patients) had a non-functional catheter $(p=1.0)$.

\section{Discussion}

Patients with ESRD must be treated with renal replacement therapies, such as haemodialysis or CAPD. CAPD increases the quality of life as it is relatively easy to use, cheaper and less

\begin{tabular}{|c|c|c|c|}
\hline & Laparoscopic & Open & p-value \\
\hline \multicolumn{4}{|c|}{ Post-operative pain scores } \\
\hline Day 0 & 8.0 & 8.7 & 0.006 \\
\hline Day 1 & 6.0 & 6.8 & 0.073 \\
\hline Day 2 & 4.7 & 5.1 & 0.093 \\
\hline Day 3 & 3.5 & 3.9 & 0.066 \\
\hline Day 7 & 2.3 & 2.5 & 0.130 \\
\hline \multicolumn{4}{|l|}{ Outcome } \\
\hline Readmission & 10 & 10 & - \\
\hline Leakage & 2 & 3 & 1.0 \\
\hline Mortality & 1 & 0 & 1.0 \\
\hline Catheter Migration & 3 & 4 & 1.0 \\
\hline Peritonitis & 4 & 6 & 0.48 \\
\hline Catheter site infection & 3 & 1 & 0.1 \\
\hline \multicolumn{4}{|l|}{ Status at 6 weeks } \\
\hline Non-functional & 2 & 3 & - \\
\hline Functional & 23 & 22 & 1.0 \\
\hline
\end{tabular}


invasive. For these reasons, approximately 1,20,000 patients use renal replacement with CAPD worldwide (2).

A successful PD programme is dependent on the proper placement of a permanent CAPD catheter, which can be placed by various techniques, including open, percutaneous and laparoscopic. Several studies have found laparoscopic PD catheter placements in wider use with satisfactory success rates and acceptable morbidity in recent years (3). Although some authors have found catheter survival to be better when placed via a laparoscope, the benefit of laparoscopic techniques remains debated. A meta-analysis by Sakurada et al. (4) found no significant advantage in outcomes, such as complication rates, catheter survival rate, pain scores or length of stay in studies comparing laparoscopic versus open CAPD catheter insertion.

In our study, the two groups were well matched regarding age, sex distribution and BMI. BMI of the patient is a major determinant in outcomes of any surgical procedure. Patients with BMI $>30 \mathrm{~kg} / \mathrm{m}^{2}$ are generally recommended to lose weight before any elective surgery to reduce the chances of postoperative complications. In the case of ESRD patients requiring CAPD catheter placement, such an option is not feasible. Patients must undergo the surgical procedure at whatever BMI they present. The mean BMI in the laparoscopic group was $25.9+/-1.41 \mathrm{~kg} / \mathrm{m}^{2}$ and that in the open group was $25.4+/-1.2 \mathrm{~kg} / \mathrm{m}^{2}(\mathrm{p}=0.177)$. These results are similar to those of van Laanen et al. (5) and Wright et al. (6).

In our study, despite a significantly larger number of patients in the laparoscopic group with a history of prior abdominal surgeries, there was no statistically significant difference in outcome regarding functional status or surgical complications between the two groups. A similar study by van Laanen et al. (5) with 23 patients (52\% of total) in the open group and 22 (48\% of total) in the laparoscopic group with a history of previous abdominal surgeries and another study by Wright et al. (6), with 5/24 patients in the open group and 11/21 patients in the laparoscopic group with a history of previous abdominal operations have shown comparable functional outcomes between the two groups. Our study population was similar to that in the study by Wright et al. (6) because there was a higher proportion of patients in the laparoscopic group (10/25-40\%) who had a history of previous abdominal surgeries compared with the open group, which had only $3 / 25(12 \%)$ patients with previous abdominal surgeries. Despite the significant difference in this parameter, there was no effect on the primary outcome measure. Although the study is not powered to analyse the comparison of the outcomes of different techniques used to place CAPD catheters in patients with a history of abdominal surgeries, a history of previous abdominal surgeries seems not to reduce success rate of laparoscopic CAPD catheter placement. The technique of CAPD catheter placement by laparoscopy has been advocated at many centres for patients with a history of abdominal surgery. The logic is that laparoscopic visualisation of the intrabdominal milieu helps the surgeon place the catheter tip correctly at a site away from adhesions and perform division of adhesions that may interfere with the proper functioning of the CAPD catheter.

In our study, both groups had $8 \%$ of patients who had previously undergone CAPD catheter placement. They had undergone catheter removal for various reasons, like outflow obstruction, peritonitis or other problems. In the study by van Laanen et al. (5), 16\% of the patients undergoing laparoscopic or open CAPD catheter insertion had a history of CAPD catheter insertion. In their study, this group of patients with a history of prior implantation of CAPD catheter had a success rate of $83 \%$ in the open group and $88 \%$ in the laparoscopic group making it a statistically insignificant parameter. Our study population had a very small proportion of patients with such a history. The patients in the laparoscopic group who had a previous history of CAPD catheter insertion had functional CAPD catheters at six weeks. In the open group, of the two patients, one patient had a non-functional CAPD catheter at six weeks. The difference between the two groups was not statistically significant.

For postoperative pain scores measured on $D+0, D+1, D+2$, $D+3$ and $D+7$, there was no significant difference in the scores between the two arms in our study except on the postoperative evening when the patients in the laparoscopic arm had significantly less pain than those in the open surgical placement arm ( $p=0.006)$. Similar to the studies by Wright et al. (6) and Jwo et al. (7), postoperative pain and the requirement for analgesics did not differ between the laparoscopic and open groups. A likely explanation is that the pain caused by the limited dissection via the small incision in the open group was equivalent to the mild pain produced by the carbon dioxide pneumoperitoneum during the laparoscopic procedure.

The incidence of catheter tip migration is reported as $2.7 \%$ and $15.0 \%$ for various catheter insertion techniques (7). We had $12 \%$ and $16 \%$ catheter tip migration rates in the laparoscopic and open surgical techniques. In our results, the open group's catheter tip migration rate was comparable to that of Jwo et al. (7), but our rate in the laparoscopic arm was higher than theirs. Studies by Soontrapornchai and Simapatanapong (3) have demonstrated a catheter tip migration rate of $0 \%$. However, in this study, fixation of the catheter to the parietal peritoneum was performed, which probably was the reason for the zeromigration rate. We did not perform this fixation suture in the laparoscopic technique as suture fixation is not an option in the open surgical technique. We wanted to compare the outcomes between the two arms without a significant difference in the technique. 
In the literature, the dialysate fluid leakage incidence after open or laparoscopic catheter placement has been reported to be between $5 \%$ and $13 \%(8,9)$. Paramedian placement, oblique catheter course through the abdominal wall, long extraperitoneal tunnel and long duration from catheter placement to dialysis initiation seem to reduce the incidence. Yun et al. (10) reported that minimising the leakage risk was minimised by reducing the trocars' number and size. In our study in place of the $10 \mathrm{~mm}$ port, we used an $8 \mathrm{~mm}$ port (that did not exceed the catheter cuff diameter) to tunnel in the catheter to reduce fluid leakages, tunnel infection and minimise the incisional hernia rate. We think that loose pericatheter tissue may increase the risk of leakage and catheter infection. Our study had a pericatheter leakage rate of $8 \%$ and $12 \%$ in the laparoscopic and open surgery arms, respectively. These results are much higher than the rates mentioned in the studies by Soontrapornchai and Simapatanapong (3) and most recently by van Laanen et al. (5). They had leakage rates of $2 \% / 2 \%$ and $2 \% / 0 \%$, respectively, in the laparoscopic and open surgery arms. Our results were closer to the rates published by Jwo et al. (7) who had leakage rates of $10 \% / 16 \%$ in the laparoscopic and open arms.

One of our patients in the laparoscopic arm died during the 6 -week follow-up period due to congestive cardiac failure. This patient did not have any surgical complications, but he died within six weeks of the procedure. Hence, he was considered a surgical mortality. Jwo et al. (7) had a mortality rate of $17.5 \%$ and $27 \%$ in the laparoscopic and open surgery arms, respectively. However, among all the mortalities in that study, only one mortality was due to catheter-related sepsis, whereas the remaining mortalities were not directly related to surgery.

The exit-site/tunnel infection incidence did not differ between the laparoscopic (12\%) and open (4\%) insertion technique in our study $(p=0.1)$. The PD catheter was subcutaneously tunnelled in all cases, which reduced the exit-site infection incidence, regardless of the insertion technique. The literature suggests a higher exit-site infection incidence in the open group 6.3\%$41 \%$ versus the laparoscopic group 2.5\%-18\% $(11,12)$.

A large case series did not report any difference in the peritonitis incidence when using the open insertion technique (2.9\%-31\%) or the laparoscopic technique (2.5\%-31\%) (1). Our study's data also showed no significant difference in the peritonitis incidence in agreement with these studies. The differences in peritonitis incidence in various reports might be partly due to differences in the prophylactic antibiotic regimens used. There is no consensus about which and when antibiotics should be administered to prevent peritonitis. The type of antibiotic used might influence the peritonitis incidence. Gadallah et al. (13) reported in a large randomized controlled trial that the use of $1 \mathrm{gm}$ vancomycin preoperatively significantly reduced the peritonitis risk compared with $1 \mathrm{gm}$ cefazolin and no antibiotic at all.

Mechanical obstructions that impair the functionality of CAPD catheters are an omental wrap, adhesions or catheter migration out of the pelvis (3). Catheter fixation to the peritoneum in the laparoscopic method might decrease the risk of this complication $(14,15)$. Gadallah et al. $(16)$ found catheter survival rates to be $77.5 \%$ and $62.5 \%$, respectively, in the laparoscopic and open groups and reported the laparoscopic group to be better. Gajjar et al. (17) showed that the functionality rate of catheters inserted by laparoscopy was $97.8 \%$, whereas it was $80 \%$ for the conventional method, with no statistical significance. In our study, the 6-week survival for catheters was 92\% in laparoscopy and $88 \%$ in open surgery group with no significant difference.

\section{Study Limitations}

A limitation of our study is that, despite randomisation, there was a statistically significant difference in patients with a previous abdominal surgery favouring the open surgery group. This could have negatively influenced the clinical success rate in the laparoscopic group. It also makes a comparison between open and laparoscopic surgery more hazardous. Larger study populations with longer follow-up are required to reach more definitive conclusions.

\section{Conclusion}

The specific advantages of open surgery are shorter operative times and more basic equipment requirements and those of the laparoscopic technique are the opportunities to do adhesiolysis and place the catheter tip in the pelvis under direct vision. Our study shows no significant difference between these two modalities regarding functional outcomes and perioperative complication rates. However, it was only a short-term outcomes study and further trials focusing on long-term outcomes are needed.

\section{Ethics}

Ethics Committee Approval: After obtaining institutional ethical committee approval (Institutional Ethics Committee, Army Hospital (R \&t R), Delhi Cantt, IEC Regn no: 93/2016).

Informed Consent: All patients for PD were included in this trial after obtaining written informed consent.

Peer-review: Externally peer-reviewed.

\section{Authorship Contributions}

Surgical and Medical Practices: A.J., G.M., Concept: R.T., G.S., Design: R.T., N.S., Data Collection or Processing: G.S., Analysis or Interpretation: N.S., Literature Search: G.S., Writing: A.J., G.S. 
Conflict of Interest: No conflict of interest was declared by the authors.

Financial Disclosure: The authors declared that this study received no financial support.

\section{References}

1. Hagen SM, Lafranca JA, Steyerberg EW, IJzermans JN, Dor FJ. Laparoscopic versus open peritoneal dialysis catheter insertion: a meta-analysis. PLoS One 2013;8:e56351.

2. Dinç B, Dinçkan A, Çiyiltepe $H$, Mesci A, Erdoğan O, Çolak T. Comparison of Laparoscopic and Conventional Methods for Continuous Ambulatory Peritoneal Dialysis Catheter Insertion in Terms of Complications. Turk Neph Dial Transpl 2012;21:156-160.

3. Soontrapornchai $P$, Simapatanapong T. Comparison of open and laparoscopic secure placement of peritoneal dialysis catheters. Surg Endosc 2005;19:137-139.

4. Sakurada T, Ueda A, Komukai D, Uchiyama K, Tsujimoto Y, Yuasa H, Ryuzaki $\mathrm{M}$, Ito $\mathrm{Y}$, Tomo $\mathrm{M}$, Nakamato $\mathrm{H}$. Outcomes after peritoneal dialysis catheter placement by laparoscopic surgery versus open surgery: systematic review and meta-analysis. Ren Replace Ther 5, 37 (2019).

5. van Laanen JHH, Cornelis T, Mees BM, Litjens EJ, van Loon MM, Tordoir JHM, Peppelenbosch AG. Randomized Controlled Trial Comparing Open Versus Laparoscopic Placement of a Peritoneal Dialysis Catheter and Outcomes: The CAPD I Trial. Perit Dial Int 2018;38:104-112.

6. Wright MJ, Bel'eed K, Johnson BF, Eadington DW, Sellars L, Farr MJ. Randomized prospective comparison of laparoscopic and open peritoneal dialysis catheter insertion. Perit Dial Int 1999;19:372-375.

7. Jwo $\mathrm{S}$, Chen $\mathrm{K}$, Lee $\mathrm{C}$, Chen $\mathrm{H}$. Prospective randomized study for comparison of open surgery with laparoscopic-assisted placement of Tenckhoff peritoneal dialysis catheter--a single center experience and literature review. J Surg Res 2010;159:489-496.
8. Leblanc M, Ouimet D, Pichette V. Dialysate leaks in peritoneal dialysis. Semin Dial 2001;14:50-54.

9. Schmidt SC, Pohle C, Langrehr JM, Schumacher G, Jacob D, Neuhaus P. Laparoscopic-assisted placement of peritoneal dialysis catheters: implantation technique and results. J Laparoendosc Adv Surg Tech A 2007;17:596-599.

10. Yun EJ, Meng MV, Brennan TV, McAninch JW, Santucci RA, Rogers SJ. Novel microlaparoscopic technique for peritoneal dialysis catheter placement. Urology 2003;61:1026-1028.

11. Strippoli GF, Tong A, Johnson D, Schena FP, Craig JC. Antimicrobial agents to prevent peritonitis in peritoneal dialysis: a systematic review of randomized controlled trials. Am J Kidney Dis 2004;44:591-603.

12. Figueiredo $A$, Goh $B-L$, Jenkins $S$, Johnson DW, Mactier R, Ramalakshmi $S$, Shrestha B, Struijk D, Wilkie M; International Society for Peritoneal Dialysis. Clinical practice guidelines for peritoneal access. Perit Dial Int 2010;30:424429.

13. Gadallah MF, Ramdeen G, Torres C, Mignore J, Patel D, Mitchell L, Tatro S. Preoperative vancomycin prophylaxis for newly placed peritoneal dialysis catheters prevents postoperative peritonitis. Adı Peri Dial 2000;16:199-203.

14. Öğünç $G$, Tuncer $M, O$ ğünç $D$, Yardimsever $M$, Ersoy F. Laparoscopic omental fixation technique vs open surgical placement or peritoneal dialysis catheters. Surg Endosc 2003;17:1749-1755.

15. Harissis HV, Katsios CS, Koliousi EL, Ikonomou MG, Siamopoulos KC, Fatouros M, Kappas AM. A new simplified one port laparoscopic technique of peritoneal dialysis catheter placement with intra-abdominal fixation. Am J Surg 2006;192:125-129.

16. Gadallah MF, Pervez A, El-Shahawy MA, Sorrells D, Zibari G, McDonald J, Work J. Peritoneoscopic versus surgical placement of peritoneal dialysis catheters: a prospective randomized study on outcome. Am J Kidney Dis 1999:33:118-122.

17. Gajjar $A H$, Rhoden $D H$, Kathuria $P$, Kaul $R$, Udupa $A D$, Jennings WC. Peritoneal dialysis catheters: Laparoscopic versus traditional placement techniques and outcomes. Am J Surg 2007;194:872-875. 